\title{
EFICIÊNCIA DA TERRA DE DIATOMÁCEA NO CONTROLE DE Sitophilus zeamais EM MILHO ARMAZENADO
}

\author{
Effectiveness of Diatomaceous Earth to Control Sitophilus \\ zeamais in Corn Stored
}

\author{
Alberto Luiz Marsaro Júnior \\ Entomologista, Doutor, Embrapa Roraima, Boa Vista - RR. E-mail: alberto@cpafrr.embrapa.br. \\ Moisés Mourão Júnior \\ Bioestatístico, Mestre, Embrapa Roraima, Boa Vista - RR. E-mail: mmourao@cpafrr.embrapa.br.
}

Wellington Robinson Soares Cizino de Paiva

Acadêmico de Farmácia da Faculdades Cathedral, Boa Vista - RR. E-mail: wellingtoncizino@hotmail.com.br.

Hosana Carolina dos Santos Barreto

Acadêmica de Química da UFRR, bolsista do programa Pibic/CNPq, Câmpus Paricarana, Boa Vista - RR. E-mail: karolina_rr@click21.com.br.

\begin{abstract}
Resumo
O gorgulho-do-milho, Sitophilus zeamais, é uma das principais pragas do milho armazenado no Brasil. O controle dessa praga é realizado principalmente com inseticidas químicos. O uso de protetores de grãos na região norte do Brasil é raro. Nesse sentido, o objetivo desta pesquisa foi avaliar a eficiência da terra de diatomácea aplicada sobre milho armazenado em Roraima, para o controle de $S$. zeamais. Grãos do híbrido BRS 3003 foram tratados com cinco dosagens de terra de diatomácea, 0, 250, 500, 750 e $1000 \mathrm{~g} / \mathrm{t}$. Cada tratamento, contendo $100 \mathrm{~g}$ de grãos, foi infestado com 30 adultos de $S$. zeamais e mantido em laboratório a $27^{\circ} \mathrm{C}$. Avaliou-se a mortalidade acumulada do $1^{\circ}$ ao $28^{\circ}$ dia. A mortalidade dos adultos foi influenciada pelas dosagens e pelo tempo de exposição dos insetos à terra de diatomácea. Modelos logísticos foram usados para descrever as curvas de mortalidade. Esses modelos foram usados para estimar o tempo necessário para obter 80, 90 e 95\% da mortalidade da população. As dosagens de 1000 e $750 \mathrm{~g} / \mathrm{t}$ alcançaram 95\% de mortalidade aos 6 e 9 dias após o tratamento, respectivamente, enquanto as dosagens de $500 \mathrm{~g} /$ t e $250 \mathrm{~g} / \mathrm{t}$ levaram 13 e 27 dias, respectivamente, para alcançar o mesmo nível de mortalidade. A terra de diatomácea na dosagem de $1000 \mathrm{~g} / \mathrm{t}$ apresentou uma alta eficiência de controle de $S$. zeamais num curto espaço de tempo.
\end{abstract}

Palavras-chave: Controle físico; Gorgulho-do-milho; Terra de diatomácea; Milho. 


\begin{abstract}
The maize weevil, Sitophilus zeamais, is one of the major pests of stored corn in Brazil. The control of this pest is realized mainly with chemical insecticides. The use of grain protectants in the north area of Brazil is rare. In this sense, the objective of this research was to evaluate the effectiveness of diatomaceous earth applied in corn stored in Roraima, to control S. zeamais. Grains of the hybrid BRS 3003 were treated with five dosages of diatomaceous earth, 0, 250, 500, 750 and $1000 \mathrm{~g} / \mathrm{t}$. Each treatment, containing $100 \mathrm{~g}$ of kernels, was infested with 30 adults of $S$. zeamais and maintained in laboratory at $27^{\circ} \mathrm{C}$. Accumulated mortality was evaluated from the $1^{\text {st }}$ to the $28^{\text {th }}$ day. The mortality of adults was influenced by the dosages and the exposure time of insects to diatomaceous earth. Logistic models were used to describe mortality curves. These models were used to estimate the necessary time to obtain 80,90 and 95\% mortality of the population. The dosages of 1000 and $750 \mathrm{~g} / \mathrm{t}$ reached $95 \%$ of mortality 6 and 9 days after treatment, respectively, while $500 \mathrm{~g} / \mathrm{t}$ and 250 $g / t$ took 13 and 27 days, respectively, to reach the same level of mortality. Diatomaceous earth in the dosage of $1000 \mathrm{~g} / \mathrm{t}$ presented a high effectiveness to control S. zeamais in a short space of time.
\end{abstract}

Keywords: Physical control; Maize weevil; Diatomaceous earth; Corn.

\title{
INTRODUÇÃO
}

O gorgulho-do-milho, Sitophilus zeamais Motschulsky (Coleoptera: Curculionidae), é uma das principais pragas do milho armazenado no Brasil. O controle desse inseto-praga é realizado principalmente por meio do uso de inseticidas químicos. Devido aos problemas de contaminação com resíduos de inseticidas nos alimentos, o uso de pós inertes para o controle de insetos de produtos armazenados tem sido bastante estudado. Existem quatro tipos básicos de pós-inertes: argilas e areias, terra de diatomáceas, sílica aerogel (silicato de sódio) e não derivados da sílica (rochas fosfatadas) (LORINI, 2001).

Dentre os pós-inertes, a terra de diatomáceas destaca-se no controle de pragas de grãos armazenados. Diversos estudos já demonstraram o potencial de controle desse produto sobre os principais insetos de armazenameto, Sitophilus granarius (L.) (Coleoptera: Curculionidae) (ALDRYHIM, 1990); Oryzaephilus surinamensis (L.) (Coleoptera: Silvanidae) (ARTHUR, 2001); Plodia interpunctella (Hubner) (Lepidoptera: Pyralidae) (MEWIS; ULRICHS, 2001); Rhyzopertha dominica (F.) (Coleoptera: Bostrichidae) (BALDASSARI et al., 2002); Prostephanus truncatus (Horn) (Coleoptera: Bostrichidae), Callosobruchus maculatus (F.) (Coleoptera: Bruchidae) and S. zeamais (STATHERS et al., 2004); Tribolium castaneum (Herbst) (Coleoptera: Tenebrionidae) (ARNAUD et al., 2005); Sitophilus oryzae (L.) (Coleoptera: Curculionidae) (ATHANASSIOU et al., 2005a); Acanthoscelides obtectus (Say) (Coleoptera: Bruchidae) (BELLO et al., 2006) e Ephestia kuehniella Zeller (Lepidoptera: Pyralidae) (COLLINS; COOOK, 2006).

A terra de diatomácea é obtida de depósitos de carapaças de algas diatomáceas oriundas da era Cenozóica, constituídas predominantemente de sílica amorfa (dióxido de sílica) (SUBRAMANYAM; ROESLI, 2000). Segundo esses autores, a morte dos insetos pela terra de diatomácea é atribuída à dessecação provocada pela adsorção e abrasividade deste pó inerte que rompe a camada de cera da epicutícula dos insetos, fazendo com que eles percam água do corpo até morrerem.

O uso de protetores de grãos na região norte do Brasil é raro. Nesse sentido, o objetivo deste trabalho foi avaliar a eficiência de terra de diatomácea, aplicada em milho armazenado no estado de Roraima, no controle de $S$. zeamais.

\section{MATERIAL E MÉTODOS}

Grãos do híbrido BRS 3001 foram tratados com cinco dosagens de terra de diatomácea ( $860 \mathrm{~g} / \mathrm{kg}$ de dióxido de sílica), 0, 250, 500, 750 e 1000 g/t, com quatro repetições. Cada tratamento, contendo $100 \mathrm{~g}$ de 
grãos, mantido em pote de vidro telado, foi infestado com 30 adultos de $S$. zeamais e mantido em condições de laboratório a $27^{\circ} \mathrm{C}$. A mortalidade acumulada foi avaliada do primeiro até o vigésimo oitavo dia após a infestação. Quando a testemunha apresentou mortalidade, as médias dos tratamentos foram corrigidas pela fórmula de Abbott (ABBOTT, 1925).

O modelo adotado para analisar os dados considerou o efeito da dosagem e o tempo de exposição da terra de diatomácea sobre a mortalidade dos insetos. Os dados de mortalidade foram submetidos à análise de variância e as médias comparadas por meio do teste de Duncan a 5\% de significância. Os dados foram analisados utilizando-se o SASâ System e o STATISTICA 5.5 (LITTEL et al., 1996; STATSOFT INC., 1999).

O comportamento de mortalidade, ao longo do tempo, foi desdobrado em cada um dos níveis marcados como significante no modelo, sob a aplicação de um ajuste não linear do tipo logístico $y=a /\left(1+\left(x / x_{0}\right)^{\mathrm{b}}\right)$. O coeficiente de determinação ajustado $\left(\mathrm{R}_{\mathrm{aj}}^{2}\right.$. foi usado como indicador de aderência do modelo em relação às observações.

\section{RESULTADOS E DISCUSSÃO}

Observou-se, neste trabalho, uma tendência de aumento da mortalidade à medida que se aumentou o tempo de exposição dos insetos à terra de diatomácea como mostra a Figura 1 e a Tabela 1. Observou-se também que a mortalidade ocorreu mais rapidamente nas dosagens mais elevadas, 750 e $1000 \mathrm{~g} / \mathrm{t}$. Na dosagem de $250 \mathrm{~g} / \mathrm{t}$, mesmo após 28 dias de exposição, não houve um controle total da população de insetos. Nessa dosagem, esse longo período de exposição necessário para causar a mortalidade dos gorgulhos pode favorecer o desenvolvimento de uma segunda geração de insetos, conforme já observado por Marsaro Júnior (2004).

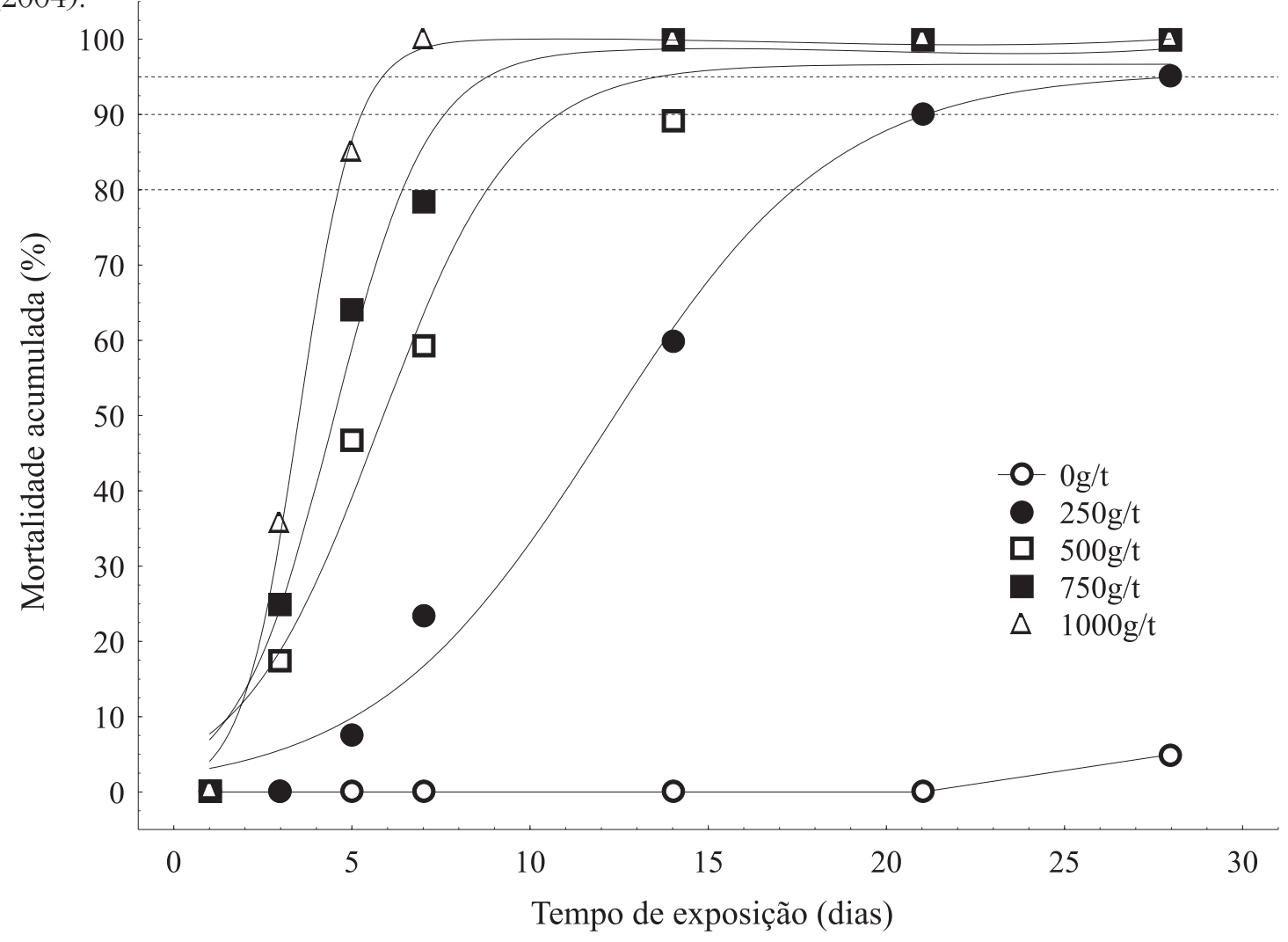

FIGURA 1 - Ajuste do modelo logístico à mortalidade acumulada em função dos dias de exposição dos insetos à terra de diatomácea

Figure 1 - Adjustment of the logistic model to the accumulated mortality in function of days of insects exposure to diatomaceous earth 
TABELA 1 - Valores médios (\%)* da mortalidade acumulada de Sitophilus zeamais em função do tempo de exposição dos insetos à terra de diatomácea e suas dosagens, bem como os coeficientes do modelo logístico ajustado.

Table 1 - Average values (\%)* of accumulated mortality of Sitophilus zeamais in function of the insects exposure time to diatomaceous earth and their dosages, as well as the parameters of the logistic model.

\section{Dosagens da terra de diatomácea $(\mathrm{g} / \mathrm{t})$}

\begin{tabular}{|c|c|c|c|c|c|c|c|c|c|c|}
\hline Dias & 0 & & 250 & & 500 & & 750 & & 1000 & \\
\hline 1 & 0,0 & $\mathrm{a}$ & 0,0 & $\mathrm{a}$ & 0,0 & $\mathrm{a}$ & 0,0 & $\mathrm{a}$ & 0,0 & $\mathrm{a}$ \\
\hline 3 & 0,0 & $\mathrm{~d}$ & 0,0 & $\mathrm{~d}$ & 17,5 & $\mathrm{C}$ & 25,0 & $\mathrm{~b}$ & 35,8 & $\mathrm{a}$ \\
\hline 5 & 0,0 & e & 7,5 & $\mathrm{~d}$ & 46,7 & $\mathrm{C}$ & 64,2 & $\mathrm{~b}$ & 85,0 & $\mathrm{a}$ \\
\hline 7 & 0,0 & $\mathrm{e}$ & 23,3 & $\mathrm{~d}$ & 59,2 & C & 78,3 & $\mathrm{~b}$ & 100,0 & a \\
\hline 14 & 0,0 & $\mathrm{~d}$ & 60,0 & c & 89,2 & $\mathrm{~b}$ & 100,0 & $\mathrm{a}$ & 100,0 & $\mathrm{a}$ \\
\hline 21 & 0,0 & c & 90,0 & $\mathrm{~b}$ & 100,0 & $\mathrm{a}$ & 100,0 & $\mathrm{a}$ & 100,0 & $\mathrm{a}$ \\
\hline 28 & 5,0 & $\mathrm{C}$ & 95,3 & b & 100,0 & $\mathrm{a}$ & 100,0 & $\mathrm{a}$ & 100,0 & $\mathrm{a}$ \\
\hline
\end{tabular}

Coeficientes e parâmetros do modelo

\begin{tabular}{llllll}
\hline & $\mathbf{0}$ & $\mathbf{2 5 0}$ & $\mathbf{5 0 0}$ & $\mathbf{7 5 0}$ & $\mathbf{1 0 0 0}$ \\
\hline a & - & 0,957 & 0,967 & 0,988 & 1,000 \\
b & - & 3,266 & 1,940 & 1,341 & 0,796 \\
x0 & - & 12,077 & 5,748 & 4,473 & 3,518 \\
R2 & - & 0,991 & 0,980 & 0,986 & 0,998 \\
R2aj. & - & 0,987 & 0,969 & 0,979 & 0,997 \\
\hline
\end{tabular}

*Valores seguidos pela mesma letra, na horizontal, não diferem estatisticamente entre si, pelo teste de Duncan ( $p \leq 0,05)$.

Pinto Júnior (1994), ao estudar a mortalidade de Sitophilus spp. expostos à terra de diatomácea nas dosagens de 250, 500 e $750 \mathrm{~g} / \mathrm{t}$, observou um efeito interativo entre a dosagem e o período de exposição, ou seja, períodos maiores de exposição, nas dosagens mais elevadas, proporcionaram um melhor controle da população, resultados que se assemelham aos encontrados nesta pesquisa.

O modelo logístico usado (TABELA 1) estimou o tempo para obter $80 \%$ de mortalidade para a dosagem de $1000 \mathrm{~g} / \mathrm{t}$ de 5 dias, para a dosagem de $750 \mathrm{~g} / \mathrm{t}$ de 6 dias, enquanto nas dosagens de 500 e 250 g/t foram necessários 9 e 17 dias, respectivamente. Para obter 95\% de mortalidade, o modelo logístico estimou o período de 6 dias para a dosagem mais elevada, um período de 9 dias para a dosagem de 750 g/t, enquanto nas dosagens de 500 e $250 \mathrm{~g} / \mathrm{t}$ seriam necessários 13 e 27 dias, respectivamente.

A alta taxa de mortalidade dos insetos, mesmo na menor dosagem, $250 \mathrm{~g} / \mathrm{t}$, também já foi observada por Arthur (2002). Utilizando a dosagem de $300 \mathrm{~g} / \mathrm{t}$, o autor verificou mortalidade superior a $82 \%$ após uma semana de exposição dos insetos à terra de diatomácea. Utilizando a dosagem de $200 \mathrm{~g} / \mathrm{t}$, Arnauld et al. (2005) verificaram mortalidade da população de T. castaneum superior a 95\%, após 21 dias de exposição.

Athanassiou et al. (2005a) também verificaram que, em baixas dosagens, para se alcançar um satisfatório nível de controle de pragas de grãos armazenados, um maior período de exposição foi necessário. Esse fato está relacionado com o modo de ação da terra de diatomácea sobre o inseto. De acordo com Subramanyam e Roesli (2000), a morte dos insetos pela terra de diatomácea é atribuída à dessecação provocada pela adsorção e abrasividade deste pó inerte que rompe a camada de cera da epicutícula dos 
insetos, fazendo com que eles percam água do corpo até morrerem. Portanto, em altas dosagens, a adsorção e a abrasividade causadas pela terra de diatomácea ocorrem mais rapidamente, causando a morte num curto intervalo de tempo, quando comparado com as menores dosagens.

Produtos compostos por terra de diatomácea usualmente necessitam de um tempo um pouco maior para matar os insetos, quando comparado com os inseticidas que agem por contato, entretanto, o efeito residual da terra de diatomácea é usualmente maior. Estudos realizados por Athanassiou et al. (2005b) demonstraram que a terra de diatomácea causou mais de $90 \%$ de mortalidade da população de $S$. oryzae em trigo tratado com esse protetor por um período superior a 270 dias.

\section{CONCLUSÕES}

A terra de diatomácea apresentou uma alta eficiência no controle de $S$. zeamais em todas as dosagens avaliadas.

Quando se aumentou a dosagem de terra de diatomácea de 0 a $1000 \mathrm{~g} / \mathrm{t}$, verificou-se que houve uma redução do tempo necessário para alcançar altos níveis de mortalidade dos insetos, sendo que para a última dosagem a mortalidade total ocorreu já no sétimo dia após sua aplicação.

\section{REFERÊNCIAS}

ABBOTT, W. S. A method of computing the effectiveness of an insecticide. Journal of Economic Entomology, v. 18, p. 265-267, 1925.

ALDRYHIM, Y. N. Efficacy of the amorphous silica dust, dryacide, against Tribolium confusum Duv. and Sitophilus granarius (L.) (Coleoptera: Tenebrionidae and Curculionidae). Journal of Stored Products Research, v. 26, p. 207-210, 1990.

ARNAUD, L. et al. Efficacy of diatomaceous earth formulations admixed with grain against of Tribolium castaneum. Journal of Stored Products Research, v. 41, p. 121-130, 2005.

ARTHUR, F. H. Immediate and delayed mortality of Oryzaephilus surinamensis (L.) exposed on wheat treated with diatomaceous earth: effects of temperature, relative humidity, and exposure interval. Journal of Stored Products Research, v. 37, p. 13-21, 2001.

Survival of Sitophilus oryzae (L.) on wheat treated with diatomaceous earth: impact of biological and environmental parameters on product efficacy. Journal of Stored Products Research, v. 38, p. 305-313, 2002.

ATHANASSIOU, C. G. et al. Insecticidal efficacy of diatomaceous earth against Sitophilus oryzae (L.) (Coleoptera: Curculionidae) and Tribolium confusum du Val (Coleoptera: Tenebrionidae) on stored wheat: influence of dose rate, temperature and exposure interval. Journal of Stored Products Research, v. 41, p. 47-55, 2005a.

Persistence and efficacy of three diatomaceous earth formulation against Sitophilus oryzae (Coleoptera: Curculionidae) on wheat and barley. Journal of Economic Entomology, v. 98, p. 1404-1412, 2005b.

BALDASSARI, N.; BALDONI, G.; BARONIO, P. Efficacy of different diatomaceous earths to control adult insects. Tecnica Molitoria, v. 53, p. 1201-1207, 2002.

BELLO, G. et al. Biocontrol of Acanthoscelides obtectus and Sitophilus oryzae with diatomaceous earth and Beauveria bassiana on stored grains. Biocontrol Science and Technology, v. 16, p. 215-220, 2006.

COLLINS, D. A.; COOK, D. A. Laboratory evaluation of diatomaceous earths, when applied as dry dust and slurries to wooden surfaces, against stored-product insect and mite pests. Journal of Stored Products Research, v. 42, p. 197-206, 2006. 
LITTEL, R. C. et al. SAS ${ }^{\circledR}$ System for Mixed Models. SAS Institute Inc.: Cary, 1996.

LORINI, I. Manual técnico para o manejo integrado de pragas de grãos de cereais armazenados. Passo Fundo: EMBRAPA Trigo, 2001.

MARSARO JÚNIOR, A. L. Resistência de genótipos de milho, Zea mays L. (Poaceae), ao ataque de Sitophilus zeamais Motschulsky (Coleoptera: Curculionidae). 2004. 84 f. Tese (Doutorado em Entomologia)-Universidade Federal do Paraná. Curitiba, 2004.

MEWIS, I.; ULRICHS, C. Action of amorphous diatomaceous earth against different stages of the stored product pests Tribolium confusum, Tenebrio molitor, Sitophilus granarius and Plodia interpunctella. Journal of Stored Products Research, v. 37, p. 153-164, 2001.

PINTO JÚNIOR, A. R. Uso de pós inertes no controle de insetos de grãos armazenados. Dissertação de Mestrado em Entomologia. 1994. 80 f. Dissertação (Mestrado em Entomologia)-Universidade Federal do Paraná. Curitiba, 1994.

STATHERS, T. E.; DENNIFF, M.; GOLOB, P. The efficacy and persistence of diatomaceous earth admixed with commodity against four tropical stored product beetle pests. Journal of Stored Products Research, v. 40, p. 113-123, 2004.

STATSOFT, INC. Statistica for Windows [Computer program manual]. StatSoft, Inc., Tulsa. 1999. Disponível em: <http://www.statsoft.com>. Acesso em: 15 de maio de 2005.

SUBRAMANYAM, B., ROELSI, R. Inert dusts. In: ; HAGSTRUM, D.W. (Ed.). Alternatives to pesticides in stored-product IPM. Norwell: Kluwer Academic Publishers, 2000. p. 321-380. 